\title{
TREnD: a Timely, Reliable, Energy-efficient and Dynamic WSN Protocol for Control Applications
}

\author{
Piergiuseppe Di Marco, Pangun Park, Carlo Fischione, and Karl Henrik Johansson
}

\begin{abstract}
Control applications over wireless sensor networks (WSNs) require timely, reliable, and energy efficient communications. Cross-layer interaction is an essential design paradigm to exploit the complex interaction among the layers of the protocol stack and reach a maximum efficiency. Such a design approach is challenging because reliability and latency of delivered packets and energy are at odds, and resource constrained nodes support only simple algorithms. In this paper, the TREnD protocol is introduced for control applications over WSNs in industrial environments. It is a cross-layer protocol that embraces efficiently routing algorithm, MAC, data aggregation, duty cycling, and radio power control. The protocol parameters are adapted by an optimization problem, whose objective function is the network energy consumption, and the constraints are the reliability and latency of the packets. TREnD uses a simple algorithm that allows the network to meet the reliability and latency required by the control application while minimizing for energy consumption. TREnD is implemented on a test-bed and compared to some existing protocols. Experimental results show good performance in terms of reliability, latency, low duty cycle, and load balancing for both static and time-varying scenarios.
\end{abstract}

\section{INTRODUCTION}

Energy efficient wireless sensor networks (WSNs) are allowing us to exploit sensing, control, and actuation via wireless communication with potential significant effects in industrial and consumer applications. Designing WSNs for the class of control applications is challenging. Traditional applications (e.g., monitoring) need a high probability of success in the packet delivery (reliability). In addition to reliability, control applications ask also for timely packet delivery (latency). If reliability and latency constraints are not met, the correct execution of control actions concerning the phenomena sensed may be severely compromised, thus creating unstable control loops [1]. The protocol design is further complicated by the need of a parsimonious use of energy [2]. High reliability and low latency may demand significant energy expenditure, thus reducing the WSN lifetime. Controllers can usually tolerate a certain degree of packet losses and delay [3]: large delays are allowed for high reliable communication, low delays are instead required if the packet loss is high. In contrast to monitoring applications, for control applications there is no need to maximize the reliability. A trade-off between reliability and latency can be exploited to minimize the energy consumption.

In this paper, we develop $\mathrm{TREnD}^{1}$, an energy-efficient

The authors are with the ACCESS Linnaeus Center, Electrical Engineering, Royal Institute of Technology, Stockholm, Sweden. E-mails: \{pidm, pgpark, carlofi,kallej\}@ee.kth.se.

This paper is supported by the EU project FeedNetBack, the Swedish Research Council, the Swedish Strategic Research Foundation, and the Swedish Governmental Agency for Innovation System.

${ }^{1}$ The acronym remarks the significant characteristics simultaneously embraced by the protocol as opposed to other solutions available from the literature: timeliness, reliability, energy efficiency and dynamic adaptation. protocol for control applications over WSNs. The distinctive property is that reliability and latency are flexible requirements incorporated in the design to boost the protocol performance.

The remainder of the paper is organized as follow: in Sec. II, we discuss the literature and highlight the original contribution. in Sec. III, we describe the system model; in Sec. IV, TREnD is presented; in Sec. V, an optimization problem is posed to optimize the protocol parameters; Sec. VI describes the protocol operation; in Sec. VII, we provide experimental results, and in Sec. VIII, we conclude the paper.

\section{BACKGROUND AND ORIGINAL CONTRIBUTION}

The standardization process for WSNs is ongoing and there is not any widely accepted complete protocol stack for WSNs [2], particularly concerning WSNs for control in industrial environments. The IEEE 802.15.4 protocol [4], which specifies physical layer and medium access control (MAC), is the base of recent solutions in industrial automation as WirelessHART and ISA100 [2]. Hence, we consider IEEE 802.15 .4 as the reference standard in our investigation.

In Tab. I, we summarize the characteristics of the main protocols for WSNs that may be used for control applications. From the table, we see that most of them (e.g., SPAN [5], GERAF [6], Dozer [7], ESRT [8], RMST [9], Flush [10], XMAC [11], MMSPEED [12]) are designed mainly for monitoring applications, since do not support simultaneously energy efficiency, reliability and latency requirements. In standard solutions for industrial applications like WirelessHART [2], reliability and security are optimized but the energy efficiency is not a critical concern since nodes are fully powered. It follows that while all these solutions offer high performance in their class of application, they are not efficiently designed for the control problems we deal in this paper.

In SERAN [13] and Breath [14] a relevant system-level design methodology has been presented for control application over WSNs. However, SERAN does not support averagehigh traffic regimes and tunable reliability requirements, which limits the performance of the protocol. Furthermore, load balancing and fair duty cycling are not taken into account in SERAN. On the other hand, Breath is limited to scenario with line topologies and source nodes at the edge of the network. We conclude that, to the best of our knowledge, there is no protocol from the literature suitable for control applications over WSNs, that is able to embrace all the techniques that concur to the energy efficiency (radio power control, MAC, routing, duty-cycling and load balancing) and, at the same time, able to guarantee reliability and latency constraints over multi-hop communication.

The original contribution of this paper is TREnD, a novel protocol for the class of industrial control applications in 
TABLE I

Protocol Comparison - The letters E, R, and L denote energy, reliability and latency. The circle denotes that a protocol is designed by considering the indication of the column, but it has not been validated experimentally. The circle with plus denotes that the protocol is designed by considering the indication and experimentally validated. The dot denotes that the protocol design does not include indication and hence cannot control it, but simulation or experiment results include it.

\begin{tabular}{|c|c|c|c|c|}
\hline Protocol & E & R & L & Layer \\
\hline SPAN [5] & $\bigcirc$ & $\cdot$ & $\cdot$ & MAC, routing \\
\hline GERAF [6] & $\bigcirc$ & & $\bigcirc$ & MAC, routing \\
\hline Dozer [7] & $\oplus$ & $\oplus$ & & MAC, routing \\
\hline ESRT [8] & & $\bigcirc$ & & MAC \\
\hline RMST [9] & & $\bigcirc$ & & MAC, transport \\
\hline Flush [10] & & $\oplus$ & & MAC \\
\hline XMAC [11] & $\oplus$ & $\cdot$ & $\cdot$ & MAC \\
\hline MMSPEED [12] & & $\bigcirc$ & $\bigcirc$ & Routing \\
\hline SERAN [13] & & & $\bigcirc$ & MAC, routing \\
\hline Breath [14] & $\oplus$ & $\oplus$ & $\oplus$ & Phy, MAC, routing \\
\hline TREnD & $\oplus$ & $\oplus$ & $\oplus$ & Phy, MAC, routing \\
\hline
\end{tabular}

clustered multi-hop WSNs. TREnD is based on a simple algorithm that allows the network to adapt dynamically to requirements variations typical of controllers. In contrast to SERAN and Breath, we adopt a novel MAC solution based on sleeping discipline and a beacon mechanism, that offers high reliability and energy efficiency, and we assume a uniform distribution of sensing nodes throughout the network. An original analysis of the performance of TREnD is provided. Finally, TREnD is completely implemented on Tmote Sky sensors [15] by using TinyOS [16].

\section{SYSTEM MODEL}

We consider a general scenario for an industrial control application: the state of a plant must be monitored at locations where electrical cabling is not available or cannot be extended, so that wireless sensor nodes are an appealing technology. The communication network must be energy efficient to guarantee long network lifetime. The sink of the network must receive packets from the single nodes with a desired probability of success and within a latency constraint demanded by the control application connected to the sink. Since control applications may need some reliability and delay during certain time interval, and a different pair of requirements during some other time interval, these reliability and latency requirements may change dynamically depending on the state of the plant and the history of the control actions.

Information taken by nodes, which are uniformly distributed in clusters, are sent to the sink node by multi-hop communication. The clustered topology is motivated by the energy efficiency, since transmitting data directly to the sink may consume more than routing through relays. The cluster formation problem has been thoroughly investigated in the literature and is out of the targets of this paper (see, e.g., [17], [18]).

In Fig. 1, the system model is reported. Nodes are deployed in an indoor environment with rooms. Each dotted curve defines a cluster of nodes. Nodes of a cluster can send packets only to the nodes of next cluster toward the controller, which takes appropriate actions upon the timely and reliable reception of source information. Hence, nodes not only send their sensed data, but also forward packets coming from clusters further away from the sink. The network controller is the sink

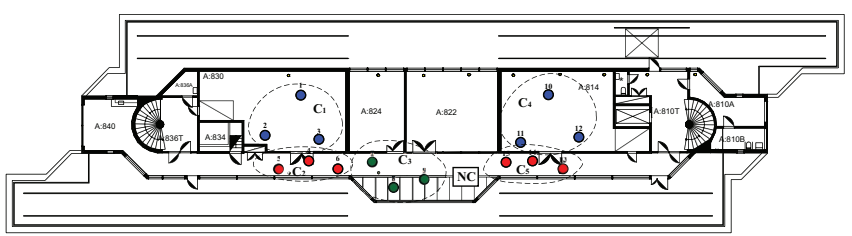

Fig. 1. Experimental Setup.

node, which, being a node of the networks, is equipped with light computing resources.

We assume that the controller knows cluster locations and the average number of nodes in each cluster, and nodes know to which cluster they belong. The controller can estimate the amount of data generated by each cluster, which is used to adapt the protocol to the traffic regime. These assumptions are reasonable in industrial environments [2].

\section{TREnD PRotocol Stack}

In this section, we introduce the protocol stack of TREnD.

Similarly to SERAN [13], the routing algorithm of TREnD is hierarchically subdivided into two parts: a static route at inter clusters level and a dynamical routing algorithm at node level. this is supported at the MAC layer by an hybrid time division multiple access and carrier sensing multiple access (TDMA/CSMA) solution.

The static schedule establishes which one is the next cluster to which nodes of a given cluster must send packets by calculating the shortest path from every cluster to the controller. The network controller runs a simple combinatorial optimization problem of latency-constrained minimum spanning tree generation [19]. Alternatively, if the number of clusters is large, the static routing schedule is pre-computed off-line for a set of cluster topologies and stored in the sink node in a look-up array. No intra cluster packet transmission is allowed.

The static routing algorithm is supported at MAC level by a weighted TDMA scheme that regulates channel access among clusters. Nodes are awake to transmit and receive only during the TDMA-slot associated to the cluster for transmission and reception, respectively, thus achieving consistent energy savings. The organization of the TDMA-cycle must consider the different traffic regimes depending on the cluster location. Since clusters closer to the sink may experience higher traffic intensity, more than one transmitting TDMA-slot can be assigned to them. It is natural to first forward packets of clusters close to the controller, since this minimizes the storage requirement in the network. To minimize the global forwarding time, the evacuation of packets of a cluster is scheduled pathby-path. By following these rules, the controller is able to generate an appropriate TDMA scheduling table.

The dynamic routing is implemented by forwarding the packets to a node within the next-hop cluster in the path chosen at random, as proposed in [6] and [18]. In such an operation, no cluster-head node is needed within clusters, and nodes need to be aware only of the next-hop cluster connectivity. The procedure for random selection of next-hop node is performed by considering a duty cycling in the receiving cluster combined with beacon transmissions.

The communication stage between nodes during a TDMAslot is managed at MAC layer by a $p$-persistent CSMA/CA 
scheme, to offer flexibility for the introduction of new nodes, robustness to node failures, and support for the random selection of next-hop node. As we will see in Sec. VII, in hybrid TDMA/CSMA solutions the $p$-persistent MAC gives better performance than the binary exponential backoff mechanism used by IEEE 802.15.4.

MAC operations of nodes are described in the following. Each node in the transmitter cluster having a packet to be sent wakes up in CSMA-slots with probability $\alpha$ and enters in listening state. At the receiver cluster, each node wakes up with probability $\beta$ and multicasts a small length of beacon message to the nodes in the transmitter cluster. An awake node that correctly receives the beacon at the transmitter side, senses the channel and, if clean, tries to unicast its packet to the beacon sender. An acknowledgement (ACK) may conclude the communication if a retransmission mechanism is implemented. If no beacon is sent or there is a collision, the awake nodes in the transmitter cluster keep on listening in the next CSMA-slot with probability $\alpha$ or go to sleep with probability $1-\alpha$.

If we compare TREnD to SERAN [13], we see that SERAN has the drawback that nodes in the receiver cluster have to be listening for the overall TDMA-slot duration, due to a contention-based transmission of the ACKs. In TREnD, the selection of the forwarding nodes follows a random policy regulated by $\beta$. The main advantage of this novel solution is the absence of delays between packets exchange during a CSMA-slot. This allows TREnD to work with a much higher traffic regime when compared to SERAN.

TREnD offers the option of data aggregation to fairly distribute the traffic load and energy consumption among clusters. The aggregation has the advantage of reducing the number of TDMA-slots per cluster and of the traffic for clusters closer to the sink. However, packet aggregation gives significant advantages only when the traffic is sufficiently high, as we will see in Sec. VII, because nodes have to idle-listen longer to catch more than one packet per time and perform the aggregation, and idle-listening is energy inefficient.

\section{PROTOCOL OPTIMIZATION}

In this section, we pose and solve an optimization problem to select the TREnD protocol parameters by minimizing the overall energy consumption of the network under reliability and latency constraints:

$$
\begin{aligned}
\min _{S, \alpha, \beta} & E_{\mathrm{tot}}(S, \alpha, \beta) \\
\text { s. t } & R(S, \alpha, \beta) \geq \Omega \\
& \operatorname{Pr}\left[D(S, \alpha, \beta) \leq D_{\max }\right] \geq \Pi .
\end{aligned}
$$

In this problem, $E_{\text {tot }}(S, \alpha, \beta)$ is the total energy consumption of the network and $R(S, \alpha, \beta)$ is the reliability constraint and $\Omega$ is the minimum desired reliability imposed by the control application. We denote by $D(S, \alpha, \beta)$ the random variable describing the distribution of the latency, by $D_{\max }$ the maximum latency desired by the control application, and by $\Pi$ the minimum probability with which such a maximum latency should be achieved. The parameters $\Omega, D_{\max }$, and $\Pi$ are the requirements of the control application. The decision variables of the optimization problem are the TREnD parameters, namely the TDMA-slot duration $S$, the access probability $\alpha$ and the wake up probability in reception $\beta$. In the following, we develop the expressions needed in the optimization problem, and derive the solution.

\section{A. Energy Consumption}

The total energy consumed by the network over a period of time is given by the combination of two components: listening and transmitting $\operatorname{cost}^{2}$.

Listening for a time $t$ gives an energy consumption that is the sum of a fixed wake up cost $E_{\mathrm{w}}$ and a time dependent cost $E_{l} t$. The energy consumption in transmission is given by four components: beacon sending $E_{\mathrm{bc}}$, clear channel assessment $E_{\text {cca }}$, packet sending $E_{\mathrm{pkt}}$ and ACK sending $E_{\text {ack }}$.

Consider a general topology with $N$ nodes per cluster and suppose that there are $G$ paths in the static routing scheduling (recall Sec. IV). Let $h_{i}$ be the number of clusters (hops) per path, we define $h_{\max }=\max _{i=1, \ldots, G} h_{i}$. We define also $W$, as the number of listening TDMA-slots in a TDMA-cycle.

Recalling that the TDMA-cycle is $T_{\text {cyc }}=S M_{s}$ where $M_{s}$ is the number of TDMA-slots in a TDMA-cycle, we have the following result:

Claim 5.1: Given traffic rate $\lambda$, the total energy consumed in a period $T_{\text {tot }}$ is

$$
\begin{aligned}
E_{\mathrm{tot}}(S, \alpha, \beta)= & \frac{T_{\mathrm{tot}}}{\gamma S} \sum_{j=1}^{\lambda T_{\mathrm{cyc}}} j \alpha \beta E_{\mathrm{cca}}+T_{\mathrm{tot}} M_{s} \lambda\left(E_{\mathrm{pkt}}+E_{\mathrm{ack}}\right) \\
& +\beta \frac{N W T_{\mathrm{tot}}}{M_{s}}\left(\frac{E_{\mathrm{bc}}}{\delta}+\frac{E_{\mathrm{w}}}{S}+E_{l}\right)
\end{aligned}
$$

Proof: A proof is provided in [20].

\section{B. Reliability Constraint}

Considering the $p$-persistent slotted CSMA MAC and the duty cycling in reception, we have the following result:

Claim 5.2: The probability of successful transmission in a CSMA-slot while there are $k$ packets waiting to be forwarded in the cluster is

$$
p_{k}=\gamma p_{\mathrm{bc}}\left(1-(1-\alpha)^{k}\right)\left(1-p_{\mathrm{cl}}\right)^{\alpha(k-1)},
$$

where $p_{\mathrm{bc}}=\gamma N \beta(1-\beta)^{N-1}$ is the successful beacon probability and $p_{\mathrm{cl}}$ is the probability of an erroneous sensing of a node, when it competes with another node.

Proof: A proof is provided in [20].

In TREnD, a radio power control is implemented, so that the attenuation of the wireless channel is compensated by the radio power, which ensures a desired packet loss probability, as proposed in [14] and [21]. As a consequence of the power control, the channel can be abstracted by a random variable with good channel probability $\gamma$. Such a modelling has been adopted also in other related works (e.g., [13], [9]). Considering the collision probability $p_{\mathrm{cl}}$, we observe that for optimization purposes an upper bound suffices. Experimental results shows that a good upper bound is $p_{\mathrm{cl}}=0.2$.

By using Claim 5.2, we can derive the following result:

Claim 5.3: Let $V(n)=\left\{1-p_{n}, 1-p_{n+1}, \ldots, 1-p_{k}\right\}$, where $p_{n}$ is the generic term given in Eq. (5) and $A(n)=\left[a_{i, j}\right]_{M_{c}}^{S-k+n}$

\footnotetext{
${ }^{2}$ Note that the costs for the initialization of the network are negligible in the energy balance
} 
be a matrix containing all the $M_{c}$ combinations with repetition of the elements in $V(n)$, taken in groups of $S-k+n$ elements. Let $h_{\max }$ be the maximum number of hops in the network. Then, the reliability of TREnD is

$R(S, \alpha, \beta)=\left(\sum_{n=0}^{k} \frac{k-n}{k} \prod_{l=n+1}^{k} p_{l}\left(\sum_{i=1}^{M_{c}} \prod_{j=1}^{S-k+n} a_{i, j}\right)\right)^{h_{\max }}$

Proof: A proof is provided in [20].

With packet aggregation enabled, the following result holds:

Claim 5.4: Let $h_{i}$ be the number of hops in the path $i$. Let $R_{z}$ be the reliability in a single hop when $z$ packets are aggregated. The reliability of a packet that experiences $j$ hops to the controller is

$$
R_{j}^{\mathrm{ag}}(S, \alpha, \beta)=R_{j-1}^{\mathrm{ag}} r_{B_{i}-j+1},
$$

where $r_{j}=\sum_{i=1}^{j}\left(1-r_{i-1}\right) \prod_{z=1}^{j-i+1} R_{z}$, with $r_{0}=0$.

Proof: A proof is provided in [20].

If the data aggregation is disabled or the size of aggregated packets does not change significantly, then we can simplify Eq. (7) and obtain the relation in Eq. (6). The previous claims are illustrated and verified by experiments in [20].

\section{Latency Constraint}

The furthest cluster from the controller is the one experiencing the highest latency. Therefore, the latency of packets coming from such a cluster must be less than or equal to a given value $D_{\max }$ with a probability $\Pi$.

Recalling that the maximum number of hops in the network is $h_{\max }$, an upper bound on the TDMA-slot duration $S$ is $S_{\max }=D_{\max } / h_{\max }$. Then, we can provide the following result:

Claim 5.5: The latency constraint in Eq. (3) is well approximated by

$$
\begin{aligned}
& \operatorname{Pr}\left[D(S, \alpha, \beta) \leq D_{\max }\right] \approx 1-\frac{1}{2} \operatorname{erfc}\left(\frac{A-\mu}{\sigma}\right) \\
& \text { where } \mathrm{A}=\left\{\begin{array}{cc}
S & \text { if } \quad S \leq \frac{D_{\max }}{h_{\max }} \\
D_{\max }-\left(h_{\max }-1\right) S & \text { if } \quad S>\frac{D_{\max }}{h_{\max }}
\end{array}\right. \\
& \mu=\sum_{\text {Proof: A proof is provided in [20]. }}^{k} 1 / p_{j} \text {, and } \sigma^{2}=\sum_{j=1}^{k}\left(1-p_{j}\right) / p_{j}^{2} .
\end{aligned}
$$

\section{Protocol Optimization}

In the previous subsection, we have established the expressions of the energy consumption in Eq. (4), the reliability in Eq. (7) and the latency constraint in Eq. (8). We observe that all this expressions are highly non-linear in the decision variables. Sensor nodes are not equipped with a high processing capacity to use these equations, therefore, we provide a computationally affordable sub-optimal solution to the optimization problem. In the following, we show that such a strategy still gives satisfactorily results.

First, we provide an empirical result on the access probability $\alpha$ and wake up probability $\beta$, for a given TDMA-slot duration $S$.
Claim 5.6: Let $N$ be the number of nodes is a cluster. Let $\lambda$ be the traffic rate, the access probability $\alpha^{*}$ and the wake up probability $\beta^{*}$, that optimize the reliability in Eq. (6), are

$$
\alpha^{*}=\frac{c_{1}}{\lambda S M_{s}+c_{2}} \quad \beta^{*}=\frac{1}{N} .
$$

with coefficients $c_{1}=2.17, c_{2}=1.81$.

Proof: A proof is provided in [20].

We note here that such choices are sub-optimal because are limited to strategies with constant access and wake up probabilities per each node.

By using Eq. (9) for the access probability and wake up probability, and by assuming $S$ as a real-valued variable, we can show that $E_{\text {tot }}$, given in Eq. (4), is a convex and monotonically decreasing function of $S$. It follows that a simple solution for the TDMA-slot duration, $S^{*}$, is given by the maximum integer value of $S$ that satisfies one out of the two constraints in the problem (1), which are given explicitly by Eqs. (7) and (8), respectively. The search of the optimal $S$ can be done by a simple additive increasing multiplicative decreasing algorithm, which we initialize by observing that $S^{*} \leq S_{\max }$. Indeed, as shown in Sec. V-C, the maximum latency requirement $D_{\max }$ provides an upper bound for $S$, given by $S_{\max }=D_{\max } / h_{\max }$.

\section{PRotocol Operation}

Suppose that the network user deploys a WSN of nodes implementing the TREnD protocol, and sets the desired control application requirements $\Omega, D_{\max }$ and $\Pi$. During an initial phase of operation the sink node retrieves the traffic and the cluster topology by the received packets. After computing or reading from a look-up array the static routing schedule and TDMA-cycle, the sink computes the optimal parameters as described in Sec. V-D. Then, the sink communicates these values to the nodes of the network by tokens. Such a token passing procedure ensures synchronization among nodes and allows for initializing and self configuring of the nodes to the optimal working point of the protocol. The token are then forwarded by the nodes closer to the sink to other nodes of the clusters far away by using the ACK mechanism described in [13]. Such tokens need also to be updated so that our protocol adapts dynamically to new nodes added in the clusters, variations in the source traffic, control application requirements, and time drift of the clocks. We experienced that a 20 TDMA-cycles period for the refreshing procedure gives satisfactory performance to maintain an optimal network operation with negligible extra energy consumption.

\section{EXPERIMENTAL IMPLEMENTATION AND VALIDATION}

In this section, we present a complete implementation of TREnD by using TinyOS 2.x [16] and Tmote Sky nodes [15]. In order to benchmark our protocol, we implemented also SERAN [13] and IEEE 802.15.4 [4]. Recall that IEEE 802.15.4 is the base for WirelessHart and other protocols for industrial automation, and that there is no other protocol available from the literature that is energy efficient, and ensures reliable and timely packet transmission, as we summarized in Tab. I. We used the default MAC parameters of IEEE 802.15.4 
so that the protocol fits in the higher level TDMA structure and routing algorithm of SERAN and TREnD.

We reproduced the reference test-bed topology reported in Fig. 1, where clusters are placed in an indoor environment. Each cluster is composed by 3 sensors, deployed at random within a circle with one meter radius. We analyze different scenarios with different sets of traffic rate $\lambda$ and control application requirements $\left(\Omega, D_{\max }\right.$, and $\left.\Pi\right)$, which we report in Tab. II. For each scenario, Tab. II shows also the optimal TDMA-slot duration, access and wake up probabilities as obtained by the optimization in Sec. V-D. We measured the duty cycle of nodes as indicator of the energy efficiency.

\section{A. Performance Comparison}

In the first set of experiments, we show the performance improvements in TREnD, when compared to SERAN. In Fig. 2, the reliability is reported as function of the traffic rate $\lambda$, by fixing $\Omega=\Pi=95 \%$, and $D_{\max }=3,9$ s. TREnD has high reliability for all traffic rate conditions and SERAN is significantly outperformed. In particular with $D_{\max }=3 \mathrm{~s}$, as traffic rate increases over $\lambda=0.3 \mathrm{pkt} / \mathrm{s}$, the reliability of SERAN significantly decreases.

In Fig. 3 we compare the energy consumption of the two protocols, showing the average duty cycle of each node, for fixed $\Omega=\Pi=95 \%, D_{\max }=3 \mathrm{~s}$ and $\lambda=0.3 \mathrm{pkt} / \mathrm{s}$. As discussed above, in this operative condition both SERAN and TREnD meet the reliability and latency constraints. By implementing TREnD with data aggregation, we observe a more balanced duty cycle among clusters, particularly for the last hop clusters. However, the price to pay for having a better load balancing is a slight increasing of the average duty cycle. In fact, TREnD presents a slightly higher duty cycle for most of the nodes, but it reduces of about $30 \%$ the energy consumption for nodes 7,8 and 9 (cluster $C_{3}$ ), which are critical for the network operation since they also forward information from clusters $C_{1}$ and $C_{2}$. This suggests that packet aggregation is a viable choice only for the clusters supporting high traffic, as those next to the sink. Hence, it is recommended to implement packet aggregation only for those clusters, while for the others no aggregation is needed. In conclusion, TREnD ensures higher reliability, load balancing and a longer network lifetime than SERAN, without any significant difference in the complexity of the scheme.

Given these results, in the following performance evaluation of TREnD we disregard SERAN and consider IEEE 802.15.4. We present two sets of experimental results, evaluated for scenarios $\mathcal{L}$ and $\mathcal{H}$ as specified in Tab. II. Fig. 4 reports the average values of reliability, latency and duty cycle as achieved by the experiments for TREnD and IEEE 802.15.4. Data of clusters belonging to the same paths are joined by lines. We see that TREnD always ensures the satisfaction of the reliability and latency constraints specified in Tab. II. TREnD guarantees much better reliability, in particular for cluster $C_{1}$ (3 hops). In fact in $C_{1}$, IEEE 802.15.4 does not fulfill the requirement. The average latency of IEEE 802.15.4 is slightly lower than TREnD, but observe that the latency of IEEE 802.15.4 is computed only for packets arriving successfully at the sink. We observe similar behavior also for other scenarios.
TABLE II

APPLICATION REQUIREMENTS AND EXPERIMENTAL RESULTS

\begin{tabular}{|c|c|c|c|c||c|c|c|}
\hline Scenario & $\lambda$ & $D_{\max }$ & $\Pi$ & $\Omega$ & $S^{*}$ & $\alpha^{*}$ & $\beta^{*}$ \\
\hline $\mathcal{L}$ & $0.1 \mathrm{pkt} / \mathrm{s}$ & $9 \mathrm{~s}$ & $95 \%$ & $95 \%$ & $3.3 \mathrm{~s}$ & 0.41 & 0.33 \\
\hline $\mathcal{H}$ & $0.3 \mathrm{pkt} / \mathrm{s}$ & $3 \mathrm{~s}$ & $95 \%$ & $95 \%$ & $1.2 \mathrm{~s}$ & 0.43 & 0.33 \\
\hline
\end{tabular}

Finally, we present some results about the duty cycle. According to the traffic load supported by the clusters and their allotted TDMA time slots, we observe that the duty cycle depends on the number of times a cluster wakes up for the forwarding procedure. The duty cycle is the same for the clusters far away from the sink $\left(C_{1}\right.$ and $C_{4}$, see Fig. 1), but for all other clusters TREnD gives a consistent reduction of the duty cycle with respect to IEEE 802.15.4.

We remark here that the duty cycle strongly depends on the traffic load in the network. In Dozer [7], an average duty cycle $0.2 \%$ is achieved for a network of 40 nodes with a packet generation period of $120 \mathrm{~s}$ each (total traffic load $\simeq 0.3 \mathrm{pkt} / \mathrm{s}$ ). TREnD gives an average duty cycle $2.5 \%$, but the total traffic load is much higher $(\simeq 5 \mathrm{pkt} / \mathrm{s})$ than Dozer.

\section{B. Dynamic Adaptation}

In the previous section, we used a static network topology where each node is placed at fixed position and the application requirements do not change with time. In this section, we show the dynamical behavior of the protocol. As we discussed before, no protocol in literature allows for a dynamical adaptation of the parameters to the application requirements.

We present the experimental results of dynamic changes between two scenarios ( $\mathcal{L}$ and $\mathcal{H}$ in Tab. II) in static and time-varying channel conditions. A Rayleigh fading channel is obtained by moving the nodes around their initial position and also by placing metal obstacles in front of the source nodes so that the line-of-sight with the sink is lost. The network starts with scenario $\mathcal{L}$ and static channel, then after 20 TDMAcycles we introduce a Rayleigh fading channel which persists until the TDMA-cycle 60. At TDMA-cycle 40, the application requirements change to scenario $\mathcal{H}$.

Figs. 5 and 6 report the resulting snapshot of the experiment in terms of reliability and latency. The reliability is measured at the sink node as average on each TDMA-cycle, while the latency is measured for each successfully received packet. In Fig. 5, we observe that TREnD guarantees the reliability requirement for both static and Rayleigh fading conditions, continuously adapting to the severe fading. The protocol is also robust to the change of scenario at TDMA-cycle 40. In Fig. 6 a snapshot of the latency is reported for clusters at different hops to the controller. We observe that the peaks of delay are limited due to the TDMA structure, the average and dynamics of the delay are slightly increasing in the time-varying stage but the latency constraint is fulfilled. Moreover, the protocol adapts well to the change of scenario at TDMA-cycle 40.

\section{CONCLUSIONS}

We proposed TREnD, a novel cross-layer protocol for control applications over WSNs, which satisfies application requirements on reliability and latency while minimizing energy consumption. We posed and solved an optimization problem 


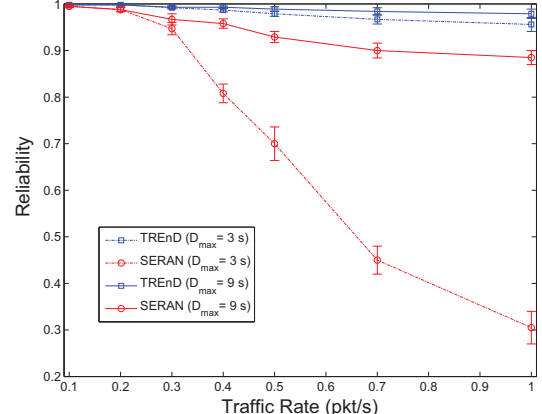

Fig. 2. TREnD and SERAN: reliability vs. traffic rate $\lambda$, for $\Omega=\Pi=95 \%$.

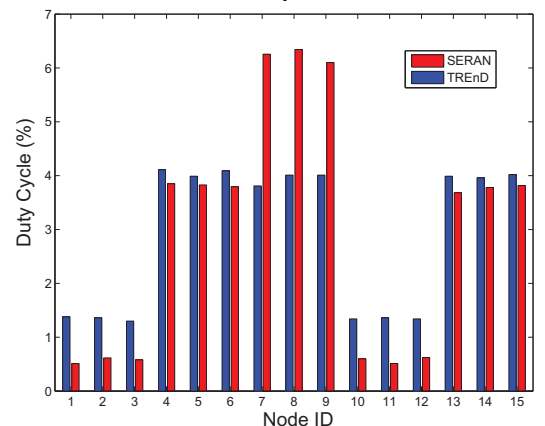

Fig. 3. TREnD and SERAN: duty cycle distribution among nodes for $\lambda=$ $0.3 p k t / s, \Omega=\Pi=95 \%$.
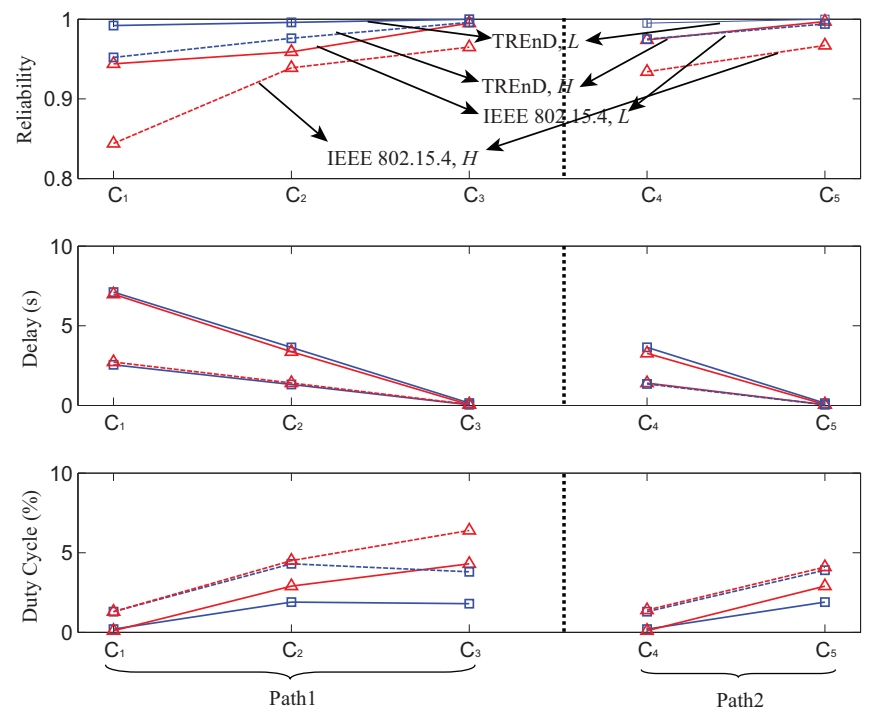

Fig. 4. TREnD and IEEE 802.15.4: reliability, latency and duty cycle for scenarios $\mathcal{L}$ and $\mathcal{H}$.

to select the protocol parameters by a simple algorithm. The experimental results showed that TREnD achieves reliable and timely transmission of packets, ensuring low node duty cycle and load balancing. Moreover, TREnD outperformed some existing solutions in terms of reliability and energy efficiency.

\section{REFERENCES}

[1] J. P. Hespanha, P. Naghshtabrizi, and Y. Xu, "A survey of recent results in networked control systems," Proceedings of the IEEE, 2007.

[2] A. Willig," "Recent and Emerging Topics in Wireless Industrial Communication," IEEE Trans. on Industrial Informatics, vol. 4,2, May 2008.

[3] W. Zhang, M. S. Braniky, S. M. Phillips, "Stability of Networked Control Systems," IEEE Control Systems Magazine, 2001.

[4] IEEE 802.15.4, IEEE, 3 Park Avenue, New York, USA, 2006. [Online]. Available: http://www.ieee802.org/15/pub/TG4.html.

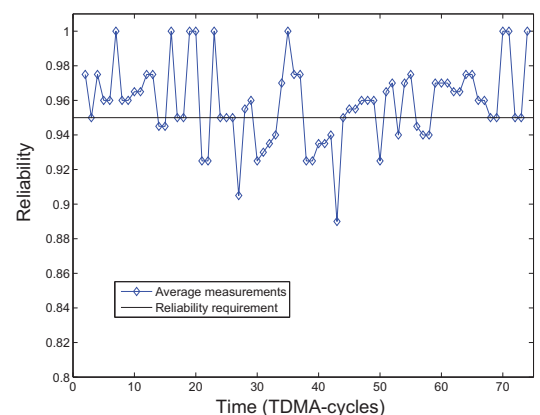

Fig. 5. TREnD: Reliability trace given by the experiments.

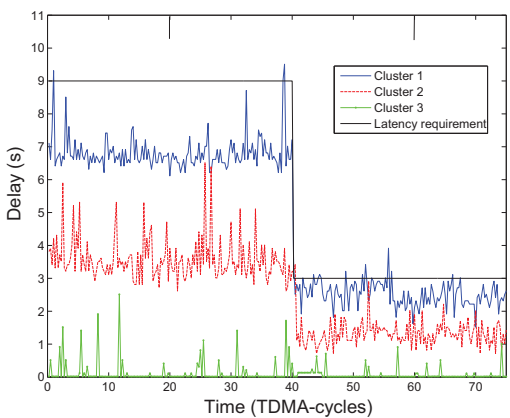

Fig. 6. TREnD: Latency trace given by the experiments.

[5] B. Chen, K. Jamieson, H. Balakrishnan, and R. Morris, "Span: An Energy-Efficient Coordination Algorithm for Topology Maintenance in Ad Hoc Wireless Networks," MobiCom, 2001.

[6] M. Zorzi and R. Rao, "Energy and Latency Performance of Geographic Random Forwarding for Ad hoc and Sensor Networks," IEEE WCNC'03.

[7] N. Burri, P. von Rickenbach, and R. Wattenhofer, "Dozer: ultra-low power data gathering in sensor networks," in ACM IPSN 2007, 2007.

[8] O. B. Akan and F. Akyildiz, "Event-to-Sink Reliable Transport in Wireless Sensor Networks," IEEE Trans. on Networking, vol. 13, 2005.

[9] F. Stann and J. Heidemann, "RMST: Reliable Data Transport in Sensor Networks," in IEEE SNPA, 2003.

[10] S. Kim, R. Fonseca, P. Dutta, A. Tavakoli, D. Culler, P. Levis, S. Shenker, and I. Stoica, "Flush: a reliable bulk transport protocol for multihop wireless networks," in ACM SenSys 2007, 2007.

[11] E. A. M. Buettner, G. Yee and R. Han, "X-MAC: A Short Preamble MAC Protocol For Duty-Cycled Wireless Sensor Networks," in 4th ACM Conference on Embedded Sensor Systems (SenSys), November 2006.

[12] E. Felemban, C.-G. Lee, and E. Eylem, "MMSPEED: Multipath MultiSPEED Protocol for QoS Guarantee of Reliability and Timeliness in Wireless Sensor Networks," IEEE Trans. on Mobile Computing, vol. 5, no. 6, June 2006

[13] A. Bonivento, C. Fischione, L. Necchi, F. Pianegiani, and A. Sangiovanni-Vincentelli, "System Level Design for Clustered Wireless Sensor Networks," IEEE Trans. on Industrial Informatics, August 2007.

[14] P. Park, C. Fischione, A. Bonivento, K. H. Johansson, and A. Sangiovanni-Vincentelli, "Breath: a Self-Adapting Protocol for Wireless Sensor Networks," IEEE SECON, June 2008.

[15] Tmote Sky Data Sheet, Moteiv, 2006. [Online]. Available: http://www.moteiv.com/products/docs/tmote-sky-datasheet.pdf

[16] D. Gay, P. Levis, and D. Culler, "Software Design Patterns for TinyOS," LCTES, 2005.

[17] O. Younis and S. Fahmy, "HEED: A Hybrid, Energy-efficient, Distributed Clustering Approach for Ad Hoc Sensor Networks," IEEE Trans. on Mobile Computing, vol. 3, no. 4, Oct.-Dec. 2004.

[18] W. Heinzelman, A. Chandrakasan, and H. Balakrishnan, "An Application-Specific Protocol Architecture for Wireless Microsensor Networks," IEEE Trans. on Wireless Communications, Oct. 2002.

[19] C. Oliveira and P. Pardalos, "A Survey of Combinatorial Optimization Problems in Multicast Routing," Computers and Operations Research, Aug. 2005.

[20] P. Di Marco, P. Park, C. Fischione, and K. H. Johansson, "A Cross-Layer Protocol for Wireless Sensor Networks in Control and Automation," Royal Institute of Technology (KTH), Tech. Rep., August 2009.

[21] M. Zuniga, B. Krishnamachari, "Analyzing the transitional region in low power wireless links," IEEE SECON, 2004. 